\title{
Dietary patterns and risk of gastric cancer: a case-control study in Uruguay
}

\author{
Eduardo De Stefani ${ }^{1}$, Pelayo Correa ${ }^{2}$, Paolo Boffetta ${ }^{3,4}$, Hugo Deneo-Pellegrini ${ }^{1}$, Alvaro L. Ronco ${ }^{1}$, \\ and María Mendilaharsu ${ }^{1}$ \\ ${ }^{1}$ Registro Nacional de Cáncer, Avenida Brasil 3080 dep 402, Montevideo, Uruguay \\ ${ }^{2}$ Department of Pathology, Louisiana State University Medical Center, New Orleans, LA, USA \\ ${ }^{3}$ Unit of Environmental Cancer Epidemiology, International Agency for Research on Cancer, Lyon, France \\ ${ }^{4}$ Division of Clinical Epidemiology, German Cancer Research Center (DKFZ), Heidelberg, Germany
}

\begin{abstract}
Background. Gastric cancer is a frequent malignancy in the Uruguayan population. In northern counties, incidence rates reach high figures (age-standardized rates [ASR], 37.3 per 100000 men and 18.3 per 100000 women). Diet is a major determinant in gastric carcinogenesis. Because foods or food groups have the advantage over nutrients in being most directly related to dietary recommendations, we decided to conduct a case-control study on the relationships between food groups and risk of gastric cancer. For this purpose, we included 240 cases and 960 controls.

Methods. In the present study we employed three analytical approaches: (1) individual food group analysis, (2) factor analysis, and (3) analysis of empirical scores of risk. Individual analysis of food groups was performed by multiple unconditional logistic regression, with food groups being the explanatory variables. Nineteen food groups were created and categorized in tertiles according to the control distribution. Factor analysis aggregated intercorrelated foods in broader eating patterns. In this study we were able to identify three factors or patterns, arbitrarily labeled "starchy," "healthy," and "mixed." Finally, empirical scores of risk were created after examining the risk of each individual food and summing each significant item. This resulted in a risk enhancing score and a protective score. Both scores were categorized in tertiles according to the control distribution.

Results. The individual analysis of food groups showed increased risks of gastric cancer for rice, salted meat, stewed meat, white bread, potatoes, and tubers. On the other hand, raw vegetables, total fruits, legumes, and black tea were inversely associated with risk of gastric cancer. All three dietary patterns, generated by factor analysis, were significantly associated with gastric carcinoma risk. Whereas the starchy factor was directly associated with gastric cancer, the healthy and mixed patterns were strongly protective. Finally, the risk enhancing empirical score displayed an increased risk of gastric cancer (odds ratio [OR], 4.1, 95\% confidence interval [CI],
\end{abstract}

Offprint requests to: E. De Stefani

Received: January 26, 2004 / Accepted: August 4, 2004
2.6-6.6), whereas the protective score showed an important reduction in risk, of 0.38 .

Conclusion. This study displayed consistent results from three different approaches. Concerning different food groups, stewed and processed meat are rich in salt; rice, tubers, and winter squash are sources of starch; and vegetables and fruits are rich in ascorbic acid and carotenoids. All these substances have been strongly related to gastric carcinogenesis. Furthermore, this study suggests that diets rich in vegetables and fruits and with low amounts of salty and starchy foods are recommendable for the prevention of gastric cancer.

Key words Gastric cancer $\cdot$ Foods $\cdot$ Factor analysis $\cdot$ Empirical scores

\section{Introduction}

Until the mid-1950s, gastric cancer was the leading malignancy in the Uruguayan population [1]. Currently, stomach cancer occupies the fifth place in frequency, being surpassed by lung, breast, colorectal, and bladder cancers [2]. When the age-standardized rates are mapped by county, northern counties display very high values (age-standardized rates [ASR], 37.3 per 100000 men and 18.3 per 100000 women) [3]. In spite of its importance, the number of epidemiologic studies dealing with stomach cancer has been relatively scant in Uruguay. Because the treatment and prognosis of this tumor site in Uruguay has been poor, the only hope of improving the outlook is related to prevention. This, in turn, depends on precise knowledge of the etiology of gastric cancer. Because diet is a major determinant in gastric carcinogenenesis, studies of foods and nutrients have been considered of great importance in the precise identification of risk and protective constituents in foods. Moreover, foods (or food groups) have the advantage over nutrients in being most directly related to dietary recommendations [4]. 
For this reason, we decided to conduct a case-control study on food groups and their relationships with gastric cancer. Furthermore, in this study we tried to identify relevant dietary patterns which could improve prevention strategies.

\section{Subjects and methods}

In the time period 1996-2000, a case-control study on environmental factors and gastric cancer was carried out in Montevideo, Uruguay.

\section{Selection of cases}

All patients with newly diagnosed and microscopically confirmed gastric carcinomas, admitted for diagnosis and treatment in the four major hospitals of Montevideo, Uruguay, were considered eligible for this study. A total of 248 cases were identified. From this initial number, 8 patients presented with disseminated disease, which precluded interviews. These patients were excluded from the study, leading to a final total of 240 patients with gastric carcinoma (response rate, $96.8 \%)$. There were 168 men $(70 \%)$ and 72 women $(30 \%)$ afflicted with the disease. The cases were distributed by subsite as follows: cardia $(10.8 \%)$, fundus $(0.8 \%)$, body $(2.5 \%)$, lesser curvature $(6.7 \%)$, greater curvature $(0.8 \%)$, and antrum and pylorus $(78.4 \%)$. Only 63 patients $(26.3 \%)$ were classified according to the Lauren scheme; the pathology reports for the remaining cases established a diagnosis of adenocarcinoma without further specification.

\section{Selection of controls}

In the same period and in the same hospitals, 1832 patients with non-neoplastic disease (1210 men and 622 women) were considered eligible for the study. These patients presented with diseases not related to tobacco smoking or alcohol drinking, and were without recent changes in their diets. From the initial number of hospitalized patients, 98 refused the interview, leaving a final number of 1734 patients (response rate, 94.6\%). From this potential pool of controls, 960 patients were frequency-matched to the cases for age (in 10-year intevals), sex, residence (Montevideo, other counties), and urban/rural status. The control series included patients with the following diseases: eye disorders $(227$ patients; $23.6 \%)$, abdominal hernia $(207 ; 21.6 \%)$, fractures $(100 ; 10.4 \%)$, skin disease $(74 ; 7.7 \%)$, acute appendicitis $(71 ; 7.4 \%)$, injuries $(67 ; 7.0 \%)$, varicose veins $(64 ; 6.7 \%)$, hydatid cyst $(50 ; 5.2 \%)$, urinary stones $(46 ; 4.8 \%)$, blood disorders $(32 ; 3.3 \%)$ and osteoarticular diseases $(22 ; 2.3 \%)$.

\section{Interviews and questionnaire}

Both series of patients (cases and controls) were interviewed face-to-face shortly after admittance to the hospitals. All the interviews were conducted in the hospitals by two trained social workers. The questionnaire included the following sections: (1) sociodemographics, (2) self-reported height and weight 5 years before the date of the interview, (3) history of cancer in firstdegree relatives, (4) a section on occupational exposure, based on job titles, (5) a complete history of tobacco smoking, (6) a complete history of alcohol drinking, (7) a complete history of maté drinking (maté is a herbal tea derived from the herb Ilex paraguariensis; this beverage is heavily consumed by the Uruguayan population and has been linked with several sites cancer), (8) menstrual and reproductive events, and (9) a food frequency questionnaire (FFQ) on 64 food items. These food items are considered as representative of the usual Uruguayan diet and allowed the calculation of total energy intake. The FFQ has not been validated, but it was tested for reproducibility with reasonably good results. Interviewers asked about the consumption of foods 5 years before the first symptom.

\section{Definition of food groups}

The following food groups were created for the analysis: (1) red meat-beef, lamb; (2) white meat - poultry, fish; (3) processed (salted meat) meat-salami; saucisson, hot dog, ham; (4) total meat-red meat, white meat, processed meat; (5) dairy foods-cheese, butter, whole milk, ice cream; (6) eggs-boiled eggs, fried eggs; (7) desserts-milk with sugar, rice pudding, custard, marmalade, cake; (8) fat-rich foods-red meat, processed meat, eggs, dairy foods, desserts; (8) grains-rice, polenta, pasta, white bread; (9) starchy foods-rice, bread, pasta, potato, sweet potato, winter squash; (10) raw vegetables-carrot, tomato, lettuce, onion; (11) cooked vegetables-swiss chard, spinach, winter squash, cabbage, cauliflower, beetroot, zucchini, red pepper; (12) total vegetables-raw vegetables, cooked vegetables; (13) citrus fruits-orange, mandarin; (14) other fruits-apple, pear, grape, peach, plum, banana, fig, fruit cocktail; (15) total fruits-citrus fruits, other fruits; (16) total vegetables and fruits-total vegetables, total fruits; (17) tubers-potato, sweet potato; (18) pulses-kidney beans, lentils; and (19) total plant foods-total vegetables, total fruits, tubers, pulses.

\section{Definition of empirical scores}

Two empirical scores were defined after testing the significance of each food item: (1) risk enhancing score barbecued meat, stew, saucisson, hot dog, ham, butter, 
mayonnaise, rice, white bread, custard, potato, winter squash; and (2) protective score - carrot, tomato, lettuce, onion, kidney bean, orange, mandarin, grape, peach, figs, coffee, and tea.

\section{Statistical analysis}

Each food group was categorized in tertiles following the distribution of the control series. Relative risks, approximated by odds ratios (ORs), were estimated by unconditional multiple logistic regression for men, women, and both sexes together [5]. Models included the following terms: age (categorical); residence, urban/ rural status, education (categorical); body mass index (categorical); tobacco smoking (pack-years, categorical); alcohol drinking (categorical); and total energy intake (continuous). For calculations involving both sexes together, a term for sex was included in the model. Each food group was entered one at a time. Test for trend for each food group was estimated after entering the categorical term for a food group as a continuous one (ordinal).

Dietary patterns were identified by factor analysis [6]. Loaded factors were rotated by varimax transformation in order to obtain a simpler structure with greater interpretability. A positive loading indicates that the food (or food group) is directly associated with the factor, whereas a negative loading indicates an inverse association $[7,8]$. The labels for the generated factors were arbitrary and based on the interpretation of the authors. Scores for rotated dietary factors (patterns) were categorized in tertiles following the distribution of the controls.

Finally, empirical dietary scores were calculated after examining the risk of each individual food and summing each significant (at the $95 \%$ confidence interval [CI] level) item [4,9]. This method resulted in a risk enhancing score and a protective score. Both scores were categorized in tertiles, according to the distribution of the controls. Odds ratios for empirical scores were estimated after controlling for potential confounding variables, according to the model explained above. All the calculations were performed with the STATA software program [10].

\section{Results}

Distribution of cases and controls by sociodemographic variables and selected risk factors is shown in Table 1. As a result of the matched design, age, sex, residence, and urban/rural status were very similar in both series of patients. Cases were slightly less educated compared with controls, but showed similar incomes. No differences were observed among the two series of patients regarding family history of stomach cancer in firstdegree relatives. Also, body mass index was rather similar in cases and controls. On the other hand, total energy intake was much higher in cases compared with controls (OR, 3.5; 95\% CI, 2.1-5.8). Residents in the northern counties of Uruguay (a high-risk area) were associated with a non-significant and modest increase in risk (OR, 1.4; 95\% CI, 0.9-2.0). Tobacco smoking was associated with an increased risk, of 1.5 , for heavy smokers, and the $P$ value for trend was significant. Total alcohol drinking was rather similar in both series of patients, although beer intake was directly associated with risk of gastric cancer (results not shown).

Odds ratios of gastric cancer for food groups (both sexes together) are shown in Table 2. Processed (salted) meat, stewed meat, rice, white bread, all cereals, potato, all tubers, winter squash, and total starchy foods were positively associated with the risk of stomach cancer. All these associations were significant, and the higher risks were associated with high consumption of rice (OR, 2.16; 95\%, CI 1.46-3.20) and stewed meat (OR, $2.02 ; 95 \%$ CI, 1.36-2.99). On the other hand, eggs, raw vegetables, total vegetables, citrus fruits, other fruits, total fruits, total vegetables and fruits, pulses, total plant foods, coffee, and tea were inversely associated with the risk of gastric cancer. The strongest negative associations were observed for tea, raw vegetables, and citrus fruits (OR for high intake of raw vegetables, $0.38 ; 95 \%$ CI, 0.26-0.57; $P$ value for trend $<0.0001)$. Red meat, white meat (poultry plus fish), total meat, dairy foods, desserts, fat-rich foods, cooked vegetables, and pasta were not associated with risk of gastric cancer.

Odds ratios of gastric cancer, stratified by sex, are shown in Table 3. In men, salted meat, stewed meat, rice, and winter squash were directly and significantly associated with gastric cancer risk. On the other hand, raw vegetables, total vegetables, citrus fruits, other fruits, total fruits, total vegetables and fruits, total plant foods, coffee, and tea were significantly associated with reduced risk of gastric carcinoma. The remaining foods and food groups were not associated with gastric cancer risk. In women, total grains, white bread, total starchy foods, cooked vegetables, potato, and all tubers were directly associated with increased risk of gastric cancer. Furthermore, all these food groups were significantly associated with the disease. Also, salted meat displayed a moderate and non-significant increase in risk (OR, 1.70; 95\% CI, 0.9-3.4). Pasta, eggs, legumes, and black tea displayed significant reductions in risk (OR for high intake of pulses, 0.37 ; 95\% CI, 0.17-0.81). Raw vegetables and citrus fruits were associated with nonsignificant reductions in risk (OR for high consumption of citrus fruits, 0.56 ; $95 \%$ CI, 0.28-1.14).

Factor analysis allowed the identification of three dietary patterns. The first one, which we called a "starchy 
Table 1. Distribution of cases and controls by sociodemographic variables and selected risk factors

\begin{tabular}{|c|c|c|c|c|c|}
\hline Variable & Category & Cases & Controls & OR & $95 \% \mathrm{CI}$ \\
\hline Age (years) & $\begin{array}{l}30-39 \\
40-49 \\
50-59 \\
60-69 \\
70-79 \\
80-89\end{array}$ & $\begin{array}{c}4(1.7) \\
16(6.7) \\
54(22.5) \\
71(29.6) \\
72(30.0) \\
23(9.6)\end{array}$ & $\begin{array}{c}16(1.7) \\
64(6.7) \\
216(22.5) \\
283(29.5) \\
290(30.2) \\
91(9.5)\end{array}$ & NA (1) & \\
\hline Sex & $\begin{array}{l}\text { Males } \\
\text { Females }\end{array}$ & $\begin{array}{r}168(70.0) \\
72(30.0)\end{array}$ & $\begin{array}{l}672(70.0) \\
288(30.0)\end{array}$ & NA & \\
\hline Residence & $\begin{array}{l}\text { Montevideo } \\
\text { Other counties }\end{array}$ & $\begin{array}{l}132(55.0) \\
108(45.0)\end{array}$ & $\begin{array}{l}539(56.1) \\
421(43.9)\end{array}$ & NA & \\
\hline Urban/rural status & $\begin{array}{l}\text { Urban } \\
\text { Rural }\end{array}$ & $\begin{array}{r}195(81.3) \\
45(18.7)\end{array}$ & $\begin{array}{l}782(81.5) \\
178(18.5)\end{array}$ & NA & \\
\hline Education (years) & $\begin{array}{l}0-2 \\
3-5 \\
6+\end{array}$ & $\begin{array}{l}69(28.8) \\
93(38.7) \\
78(32.5)\end{array}$ & $\begin{array}{l}252(26.2) \\
326(34.0) \\
382(39.8)\end{array}$ & $\begin{array}{l}1.0 \\
1.0 \\
0.7\end{array}$ & $\begin{array}{l}0.7-1.5 \\
0.5-1.1\end{array}$ \\
\hline Income (US dollars) & $\begin{array}{l}\leqq 147 \\
149+ \\
\text { Missing data }\end{array}$ & $\begin{array}{r}95(39.6) \\
113(47.1) \\
32(13.3)\end{array}$ & $\begin{array}{l}400(41.7) \\
378(39.4) \\
182(18.9)\end{array}$ & $\begin{array}{l}1.0 \\
1.2 \\
-\end{array}$ & $\begin{array}{c}0.9-1.7 \\
-\end{array}$ \\
\hline Family history & $\begin{array}{l}\text { No } \\
\text { Yes }\end{array}$ & $\begin{array}{c}230(95.8) \\
10(4.2)\end{array}$ & $\begin{array}{c}920(95.8) \\
40(4.2)\end{array}$ & $\begin{array}{l}1.0 \\
1.0\end{array}$ & $0.5-2.0$ \\
\hline Body mass index & $\begin{array}{l}\leqq 22.8 \\
22.9-25.2 \\
25.3-27.7 \\
27.8+\end{array}$ & $\begin{array}{l}67(27.9) \\
62(25.8) \\
56(23.3) \\
55(22.9)\end{array}$ & $\begin{array}{l}242(25.2) \\
239(24.9) \\
239(24.9) \\
240(25.0)\end{array}$ & $\begin{array}{l}1.0 \\
0.9 \\
0.8 \\
0.8\end{array}$ & $\begin{array}{l}0.6-1.4 \\
0.6-1.2 \\
0.5-1.2\end{array}$ \\
\hline Total calories & $\begin{array}{l}\leqq 1696 \\
1697-2041 \\
2042-2443 \\
2444+\end{array}$ & $\begin{array}{l}23(9.6) \\
56(23.3) \\
80(33.3) \\
81(33.8)\end{array}$ & $\begin{array}{l}240(25.0) \\
240(25.0) \\
240(25.0) \\
240(25.0)\end{array}$ & $\begin{array}{l}1.0 \\
2.4 \\
3.5 \\
3.5\end{array}$ & $\begin{array}{l}1.4-4.1 \\
2.1-5.8 \\
2.1-5.8\end{array}$ \\
\hline Birthplace & $\begin{array}{l}\text { Montevideo } \\
\text { South } \\
\text { North }\end{array}$ & $\begin{array}{l}81(33.8) \\
93(38.6) \\
66(27.5)\end{array}$ & $\begin{array}{l}379(39.5) \\
364(37.9) \\
217(22.6)\end{array}$ & $\begin{array}{l}1.0 \\
1.2 \\
1.4\end{array}$ & $\begin{array}{l}0.8-1.7 \\
0.9-2.0\end{array}$ \\
\hline Tobacco smoking ${ }^{\mathrm{a}}$ & $\begin{array}{l}\text { Never smokers } \\
1-24 \\
25-50 \\
51+\end{array}$ & $\begin{array}{l}86(35.8) \\
37(15.4) \\
50(20.8) \\
67(27.9)\end{array}$ & $\begin{array}{l}358(37.3) \\
214(22.3) \\
203(21.1) \\
185(19.3)\end{array}$ & $\begin{array}{l}1.0 \\
0.7 \\
1.0 \\
1.5\end{array}$ & $\begin{array}{l}0.5-1.1 \\
0.7-1.5 \\
1.0-2.2\end{array}$ \\
\hline Alcohol drinking ${ }^{\mathrm{b}}$ & $\begin{array}{l}\text { Never drinkers } \\
1-60 \\
61-120 \\
121+\end{array}$ & $\begin{array}{r}114(47.5) \\
38(15.8) \\
29(12.1) \\
59(24.6)\end{array}$ & $\begin{array}{l}418(43.5) \\
234(24.4) \\
140(14.6) \\
168(17.5)\end{array}$ & $\begin{array}{l}1.0 \\
0.6 \\
0.8 \\
1.3\end{array}$ & $\begin{array}{l}0.4-0.9 \\
0.5-1.2 \\
0.9-1.8\end{array}$ \\
\hline Number of patients & $240(100)$ & $960(100)$ & & & \\
\hline
\end{tabular}

Values in parentheses are percentages

NA, Not applicable; CI, confidence interval

a Pack-years

${ }^{\mathrm{b}}$ Milliliters of ethanol per day

pattern", reflected the correlated intakes of foods rich in starch, such as total grains and tubers (Table 4). The second pattern or factor, called "healthy pattern", displayed relatively high positive loadings of white meat, dairy foods, desserts, raw vegetables, and fruits. The third pattern was labeled "mixed pattern" and showed relatively high loadings of red meat, processed meat, eggs, and pulses.

Odds ratios for the dietary patterns are shown in Table 5. The starchy pattern was directly associated with risk of gastric cancer, both unadjusted and adjusted for total energy in the multivariate model. There was evidence of a linear trend $\left(\chi^{2}, 14.82 ; P\right.$ value for trend, $0.0002)$. The high load for this factor displayed a high increase in risk (OR, 2.51; 95\% CI, 1.54-4.10). When the results were discriminated for sex, ORs were much higher (OR, 4.17; 95\% CI, 1.78-9.79; $P$ value for trend, 0.001). The crude ORs for the healthy pattern did not reach formal statistical significance at the $95 \%$ CI level, although the highest load showed a protective effect of 
Table 2. Odds ratios of gastric cancer for food groups; both sexes combined $\mathrm{d}^{\mathrm{a}, \mathrm{b}}$

\begin{tabular}{|c|c|c|c|c|c|}
\hline \multirow[b]{3}{*}{ Food group } & \multicolumn{5}{|c|}{ Tertiles of consumption } \\
\hline & \multicolumn{2}{|c|}{ II } & \multicolumn{2}{|c|}{ III } & \multirow[b]{2}{*}{$P$ value for trend } \\
\hline & OR & $95 \% \mathrm{CI}$ & OR & $95 \% \mathrm{CI}$ & \\
\hline Red meat & 1.05 & $070-1.56$ & 1.10 & $0.71-1.71$ & 0.67 \\
\hline Poultry & 0.73 & $0.51-1.05$ & 0.98 & $0.67-1.44$ & 0.96 \\
\hline Fish & 0.75 & $0.51-1.09$ & 0.73 & $0.51-1.03$ & 0.08 \\
\hline Salted meat & 1.32 & $0.89-1.96$ & 1.98 & $1.35-2.90$ & 0.0003 \\
\hline Stewed meat & 1.13 & $0.76-1.69$ & 1.87 & $1.25-2.79$ & 0.001 \\
\hline Total meat & 1.02 & $0.68-1.53$ & 1.19 & $0.77-1.84$ & 0.42 \\
\hline Dairy foods & 1.24 & $0.85-1.80$ & 0.89 & $0.59-1.33$ & 0.50 \\
\hline Eggs & 0.67 & $0.47-0.97$ & 0.48 & $0.33-0.69$ & 0.0001 \\
\hline Desserts & 1.23 & $0.85-1.79$ & 1.07 & $0.72-1.59$ & 0.80 \\
\hline Rice & 1.01 & $0.67-1.54$ & 2.01 & $1.34-3.00$ & 0.0002 \\
\hline White bread & 1.15 & $0.77-1.72$ & 1.53 & $1.01-2.30$ & 0.04 \\
\hline Total grains & 1.63 & $1.07-2.49$ & 1.83 & $1.17-2.85$ & 0.01 \\
\hline Starchy foods & 1.55 & $1.01-2.38$ & 1.71 & $1.08-2.72$ & 0.03 \\
\hline Raw vegetables & 0.88 & $0.62-1.23$ & 0.38 & $0.26-0.57$ & $<0.0001$ \\
\hline Cooked vegetables & 1.01 & $0.70-1.46$ & 1.01 & $0.70-1.47$ & 0.94 \\
\hline Total vegetables & 0.94 & $0.66-1.34$ & 0.60 & $0.41-0.88$ & 0.01 \\
\hline Citrus fruits & 0.84 & $0.60-1.18$ & 0.45 & $0.30-0.68$ & 0.0002 \\
\hline Other fruits & 0.46 & $0.31-0.67$ & 0.64 & $0.45-0.91$ & 0.01 \\
\hline Total fruits & 0.49 & $0.34-0.71$ & 0.51 & $0.35-0.74$ & 0.0002 \\
\hline Total vegetables and fruits & 0.59 & $0.41-0.85$ & 0.57 & $0.39-0.82$ & 0.002 \\
\hline All tubers & 1.69 & $1.13-2.52$ & 1.49 & $0.98-2.27$ & 0.10 \\
\hline Pulses & 0.93 & $0.65-1.32$ & 0.56 & $0.37-0.85$ & 0.007 \\
\hline Total plant foods & 0.74 & $0.51-1.07$ & 0.57 & $0.38-0.84$ & 0.005 \\
\hline Coffee & 0.83 & $0.58-1.18$ & 0.42 & $0.25-0.68$ & 0.0007 \\
\hline Tea & 0.43 & $0.27-0.69$ & 0.13 & $0.05-0.32$ & $<0.0001$ \\
\hline
\end{tabular}

OR, Odds ratio

${ }^{a}$ Reference category: lowest tertile of intake

${ }^{\mathrm{b}}$ Adjusted for age (categorical); sex, residence, urban/rural status, education (categorical); body mass index (categorical); and total energy intake (continuous)

$25 \%$ (results not shown). When this pattern was fully adjusted, a strong reduction in risk was evident (OR for the highest load, $0.48 ; 95 \% \mathrm{CI}, 0.32-0.71 ; P$ value for trend, 0.0002). Whereas men displayed a strong reduction in risk (OR, $0.31 ; 95 \% \mathrm{CI}, 0.19-0.52 ; P$ value for trend, $<0.0001)$, women were not associated with the healthy pattern (OR, 1.15; 95\% CI, 0.51-2.59; $P$ value for trend, 0.53). Finally, the mixed pattern was not associated with risk of stomach cancer (crude OR for the highest load, $0.99 ; 95 \%$ CI, $0.65-1.52 ; P$ value for trend, $0.98)$. When this factor was fully adjusted in the multivariate model which included total energy intake, the highest load was strongly protective (OR, $0.59,95 \%$ CI $0.38-0.90 ; P$ value for trend, 0.01$)$. This reflected the strong confounding effect of total energy. Whereas men displayed a modest reduction in risk, which was nonsignificant ( $P$ value for trend, 0.20), women were strongly associated with the healthy pattern, with an OR of 0.27 (95\% CI, 0.11-0.68; $P$ value for trend, 0.02).

Total energy intake was highly positively correlated with the three factors. The highest correlation coefficient was observed between starchy pattern and total energy (rho, 0.62), closely followed by the coefficient between mixed pattern and energy (rho, 0.53). Also, starchy and mixed patterns were significantly correlated (rho, 0.28). On the other hand, starchy and healthy patterns lacked any correlation (rho, 0.00) (results not shown in Tables).

Odds ratios of gastric cancer for empirical dietary scores are shown in Table 6. As expected, the risk enhancing score was strongly and directly associated with an increased threefold risk of stomach cancer (OR, 3.16; 95\% CI, 2.10-4.75; $P$ value for trend, $<0.0001)$. There were no significant differences by sex. On the other hand, the protective score displayed an impressive reduction in risk (OR, 0.23; 95\% CI, 0.15-0.36; $P$ value for trend, $<0.0001)$. There was no heterogeneity between sexes.

\section{Discussion}

According to the results of our study, we found significant associations between the three dietary patterns and gastric cancer risk. These associations were more 
Table 3. Odds ratios of gastric cancer, by $\operatorname{sex}^{\mathrm{a}, \mathrm{b}, \mathrm{c}}$

\begin{tabular}{|c|c|c|c|c|c|}
\hline \multirow[b]{2}{*}{ Food group } & \multicolumn{2}{|c|}{ Men } & \multicolumn{2}{|c|}{ Women } & \multirow[b]{2}{*}{$P$ value for heterogeneity } \\
\hline & OR & $95 \% \mathrm{CI}$ & OR & $95 \% \mathrm{CI}$ & \\
\hline Red meat & 1.05 & $0.63-1.75$ & 1.36 & $0.58-3.17$ & 0.36 \\
\hline White meat & 0.94 & $0.60-1.48$ & 1.17 & $0.55-2.49$ & 0.57 \\
\hline Poultry & 1.08 & $0.69-1.70$ & 0.68 & $0.33-1.38$ & 0.32 \\
\hline Fish & 0.76 & $0.50-1.17$ & 0.62 & $0.32-1.20$ & 0.63 \\
\hline Salted meat & 2.27 & $1.43-3.62$ & 1.70 & $0.85-3.40$ & 0.56 \\
\hline Stewed meat & 2.28 & $1.39-3.73$ & 1.12 & $0.53-2.38$ & 0.25 \\
\hline Total meat & 1.03 & $0.61-1.73$ & 1.70 & $0.71-4.07$ & 0.12 \\
\hline Dairy foods & 0.75 & $0.46-1.20$ & 1.45 & $0.64-3.29$ & 0.12 \\
\hline Eggs & 0.50 & $0.32-0.77$ & 0.40 & $0.19-0.83$ & 0.94 \\
\hline Desserts & 1.01 & $0.62-1.66$ & 1.13 & $0.52-2.45$ & 0.61 \\
\hline Fat-rich foods & 0.93 & $0.51-1.70$ & 0.82 & $0.32-2.11$ & 0.64 \\
\hline Rice & 2.43 & $1.48-3.98$ & 1.24 & $0.61-2.54$ & 0.24 \\
\hline Polenta & 0.56 & $0.35-0.88$ & 0.31 & $0.15-0.65$ & 0.26 \\
\hline Pasta & 1.08 & $0.62-1.88$ & 0.23 & $0.08-0.62$ & 0.02 \\
\hline White bread & 1.16 & $0.71-1.92$ & 4.14 & $1.92-8.92$ & 0.01 \\
\hline Total grains & 1.45 & $0.82-2.57$ & 4.63 & $2.07-10.3$ & 0.03 \\
\hline Starchy foods & 1.34 & $0.73-2.44$ & 4.23 & $1.88-9.53$ & 0.04 \\
\hline Raw vegetables & 0.31 & $0.19-0.51$ & 0.59 & $0.28-1.22$ & 0.08 \\
\hline Cooked vegetables & 0.77 & $0.49-1.20$ & 2.46 & $1.09-5.54$ & 0.01 \\
\hline Winter squash & 1.68 & $1.11-2.54$ & 1.58 & $0.83-3.02$ & 0.85 \\
\hline Total vegetables & 0.39 & $0.24-0.63$ & 1.57 & $0.74-3.32$ & 0.001 \\
\hline Citrus fruits & 0.41 & $0.25-0.68$ & 0.56 & $0.28-1.14$ & 0.33 \\
\hline Other fruits & 0.44 & $0.28-0.70$ & 1.13 & $0.58-2.21$ & 0.01 \\
\hline Total fruits & 0.33 & $0.20-0.53$ & 1.06 & $0.53-2.12$ & 0.002 \\
\hline Total vegetables and fruits & 0.36 & $0.22-0.58$ & 1.43 & $0.69-2.98$ & 0.001 \\
\hline Potato & 1.30 & $0.82-2.05$ & 2.85 & $1.37-5.90$ & 0.06 \\
\hline All tubers & 1.16 & $0.70-1.94$ & 2.36 & $1.09-5.12$ & 0.11 \\
\hline Pulses & 0.63 & $0.38-1.04$ & 0.37 & $0.17-0.81$ & 0.68 \\
\hline Total plant foods & 0.39 & $0.24-0.64$ & 1.37 & $0.60-3.10$ & 0.003 \\
\hline Coffee & 0.55 & $0.38-0.82$ & 0.95 & $0.54-1.67$ & 0.09 \\
\hline Tea & 0.23 & $0.13-0.41$ & 0.47 & $0.25-0.88$ & 0.10 \\
\hline
\end{tabular}

${ }^{a}$ Data indicate the highest tertile of consumption

${ }^{\mathrm{b}}$ Reference category: lowest tertile of intake

${ }^{\mathrm{c}}$ Adjusted for age (categorical); residence, urban/rural status, education (categorical); body mass index (categorical); tobacco smoking (categorical); alcohol drinking (categorical); and total energy intake (continuous)

Table 4. Factor-loading matrix for the three major dietary patterns found in the study population

\begin{tabular}{lrrr}
\hline Food group & Factor 1 (starchy) & Factor 2 (healthy) & Factor 3 (mixed) \\
\hline Red meat & 0.26 & -0.06 & 0.39 \\
White meat & -0.06 & 0.31 & -0.21 \\
Processed meat & 0.07 & 0.15 & 0.39 \\
Dairy foods & 0.14 & 0.32 & 0.03 \\
Eggs & 0.16 & 0.08 & 0.26 \\
Desserts & 0.02 & 0.41 & 0.11 \\
Total grains & 0.39 & -0.08 & 0.11 \\
Raw vegetables & -0.08 & 0.39 & 0.05 \\
Cooked vegetables & 0.34 & 0.37 & 0.02 \\
Citrus fruits & 0.05 & 0.26 & 0.17 \\
Other fruits & -0.05 & 0.46 & -0.03 \\
Tubers & 0.57 & 0.04 & 0.09 \\
Pulses & 0.02 & 0.21 & 0.31 \\
Coffee & 0.03 & 0.21 & 0.16 \\
Tea & -0.30 & 0.16 & 0.02 \\
Soft drinks & -0.08 & 0.14 & 0.03 \\
Maté & 0.21 & -0.12 & 0.22 \\
Alcohol & 0.17 & -0.13 & 0.29 \\
\hline
\end{tabular}


Table 5. Odds ratios of gastric cancer for dietary patterns ${ }^{\mathrm{a}}$

\begin{tabular}{|c|c|c|c|c|c|c|c|}
\hline \multirow{2}{*}{$\begin{array}{l}\text { Factor } \\
\text { Starchy foods }\end{array}$} & \multirow[b]{2}{*}{ Cases/Controls } & \multicolumn{2}{|c|}{ Men } & \multicolumn{2}{|c|}{ Women } & \multicolumn{2}{|c|}{ Both sexes } \\
\hline & & OR & $95 \% \mathrm{CI}$ & OR & $95 \% \mathrm{CI}$ & OR & $95 \% \mathrm{CI}$ \\
\hline Low & $35 / 320$ & 1.0 & & 1.0 & & 1.0 & \\
\hline Medium & $88 / 320$ & 1.66 & $0.95-2.90$ & 3.25 & $1.49-7.08$ & 2.17 & $1.38-3.41$ \\
\hline \multirow[t]{3}{*}{ High } & $117 / 320$ & 1.89 & $1.03-3.45$ & 4.17 & $1.78-9.79$ & 2.51 & $1.54-4.10$ \\
\hline & $P$ value for trend & 0.05 & & 0.001 & & 0.0007 & \\
\hline & & \multicolumn{2}{|c|}{ Men } & \multicolumn{2}{|c|}{ Women } & \multicolumn{2}{|c|}{ Both sexes } \\
\hline Healthy & Cases/Controls & OR & $95 \% \mathrm{CI}$ & OR & $95 \% \mathrm{CI}$ & OR & $95 \% \mathrm{CI}$ \\
\hline Low & $96 / 320$ & 1.0 & & 1.0 & & 1.0 & \\
\hline Medium & $72 / 320$ & 0.61 & $0.41-0.92$ & 0.84 & $0.35-1.97$ & 0.63 & $0.44-0.91$ \\
\hline \multirow[t]{3}{*}{ High } & $72 / 320$ & 0.31 & $0.19-0.52$ & 1.15 & $0.51-2.59$ & 0.48 & $0.32-0.71$ \\
\hline & $P$ value for trend & $<0.0001$ & & 0.53 & & 0.0002 & \\
\hline & & \multicolumn{2}{|c|}{ Men } & \multicolumn{2}{|c|}{ Women } & \multicolumn{2}{|c|}{ Both sexes } \\
\hline Mixed & Cases/Controls & OR & $95 \% \mathrm{CI}$ & OR & $95 \% \mathrm{CI}$ & OR & $95 \% \mathrm{CI}$ \\
\hline Low & $70 / 320$ & 1.0 & & 1.0 & & 1.0 & \\
\hline Medium & $94 / 320$ & 1.00 & $0.63-1.60$ & 1.38 & $0.74-2.60$ & 1.09 & $0.75-1.57$ \\
\hline \multirow{2}{*}{ High } & $76 / 320$ & 0.73 & $0.43-1.22$ & 0.27 & $0.11-0.68$ & 0.59 & $0.38-0.90$ \\
\hline & $P$ value for trend & 0.20 & & 0.02 & & 0.01 & \\
\hline
\end{tabular}

${ }^{a}$ Adjusted for age (categorical); residence, urban/rural status, education (categorical); body mass index (categorical); tobacco smoking (categorical); alcohol drinking (categorical); and total energy intake (continuous)

Table 6. Odds ratios of gastric cancer for empirical dietary scores ${ }^{\mathrm{a}}$

\begin{tabular}{|c|c|c|c|c|c|c|c|}
\hline \multicolumn{8}{|c|}{ Risk enhancing score } \\
\hline \multirow[b]{2}{*}{ Servings/year } & \multirow[b]{2}{*}{ Cases/Controls } & \multicolumn{2}{|c|}{ Men } & \multicolumn{2}{|c|}{ Women } & \multicolumn{2}{|c|}{ Both sexes } \\
\hline & & OR & $95 \% \mathrm{CI}$ & OR & $95 \% \mathrm{CI}$ & OR & $95 \% \mathrm{CI}$ \\
\hline$\leqq 1411$ & $41 / 320$ & 1.0 & & 1.0 & & 1.0 & \\
\hline $1412-2162$ & $87 / 320$ & 2.05 & $1.22-3.34$ & 1.97 & $0.96-4.04$ & 2.04 & $1.35-3.09$ \\
\hline $2163+$ & $112 / 320$ & 2.81 & $1.69-4.68$ & 4.28 & $2.12-8.64$ & 3.16 & $2.10-4.75$ \\
\hline & $P$ value for trend & 0.0001 & & $<0.0001$ & & $<0.0001$ & \\
\hline
\end{tabular}

Protective score

\begin{tabular}{|c|c|c|c|c|c|c|c|}
\hline \multirow[b]{2}{*}{ Servings/year } & \multirow[b]{2}{*}{ Cases/Controls } & \multicolumn{2}{|c|}{ Men } & \multicolumn{2}{|c|}{ Women } & \multicolumn{2}{|c|}{ Both sexes } \\
\hline & & OR & $95 \% \mathrm{CI}$ & OR & $95 \% \mathrm{CI}$ & OR & $95 \% \mathrm{CI}$ \\
\hline$\leqq 391$ & $131 / 320$ & 1.0 & & 1.0 & & 1.0 & \\
\hline $392-811$ & $77 / 320$ & 0.48 & $0.32-0.72$ & 0.86 & $0.46-1.62$ & 0.57 & $0.41-0.80$ \\
\hline \multirow{2}{*}{$812+$} & $32 / 320$ & 0.20 & $0.11-0.35$ & 0.33 & $0.16-0.69$ & 0.23 & $0.15-0.36$ \\
\hline & $P$ value for trend & $<0.0001$ & & 0.004 & & $<0.0001$ & \\
\hline
\end{tabular}

a Adjusted for age (categorical); residence, urban/rural status, education (categorical); body mass index (categorical); tobacco smoking (categorical); alcohol drinking (categorical); and total energy intake (continuous)

evident after adjusting for total energy intake in a multivariate model. This is not surprising, because total calorie intake was highly correlated with these patterns. Furthermore, in our study population, energy was a strong risk factor. Thus, total energy consumption fulfils the conditions of an important confounder of eating patterns. In some studies, energy intake was reported as being directly associated with risk of stomach cancer [11].

The starchy pattern showed heavy loading of grains and tubers. Both these food groups are rich in starch, which has been considered in recent reviews as a possible risk factor for gastric cancer [12,13]. Several studies reported increased risk of this malignancy for 
rice, potato, and white bread intakes [11,14,15,16-25]. hese individual foods were included in our starchy pattern. The results for the starchy pattern were consistent with our findings for food groups and for the empirical scores. Both our factor analysis and analysis of empirical scores revealed increases in risk for white rice, bread, potato, and winter squash. Also, the prospective study of Kneller et al. [22], in the United States, reported high risks with total carbohydrates and cooked cereal. The mechanism(s) of starchy foods in gastric carcinogenesis are unclear at the present. These foods could be confounded by incomplete evaluation of socioeconomic status [26]. Another explanation has been suggested by Buiatti et al. [27]. According to these authors, starchy foods are frequently consumed after reheating, in the absence of a satisfactory reduction in their bacterial content. Also, La Vecchia et al. [26] have suggested that traditional foods are associated with a deficiency of B vitamins. Starchy foods could be associated with low-protein diets, which may favor acidcatalyzed nitrosation in the stomach because of the poor buffering capacity of such diets [13]. Finally, high-starch diets may also result in mechanical damage to the gastric mucosa [13].

The "healthy" pattern was characterized by positive loading of vegetables and fruits and, to a lesser degree, by white meat and dairy foods. According to recent reviews and previous reports [11-13,15-17, 19,20,23,24,27-31], vegetables and fruits are strongly protective in gastric cancer. Moreover, these food groups are rich, among other nutrients and bioactive substances, in ascorbic acid, beta-carotene, and other carotenoids. In particular, vitamin $\mathrm{C}$ blocks intragastric nitrosation, thus interrupting the steps towards the development of atrophic gastritis, dysplasia, and carcinoma in gastric carcinogenesis [32]. In a recent randomized trial of antioxidant supplements for the chemoprevention of gastric dysplasia, ascorbic acid, and beta-carotene reduced the progression to gastric atrophy (relative risk [RR], 0.30) and to intestinal metaplasia (RR, 0.29) [33]. These figures highlight the importance of both vitamins and their sources (vegetables and fruits) in gastric carcinogenesis. According to Key et al. [34], randomized controlled trials eliminate the biases which could be present in observational studies. Thus, the above-mentioned trial of Correa et al. [33] supports our results.

Also, at least four prospective studies have reported significant reductions in risk for vegetable and fruit consumption: for total plant foods in the United States [35], for green/yellow vegetables in Japan [36], and for total fruits and vegetables in Wales [37]. On the other hand, Botterweck et al. [38] suggested that vegetables and fruits were not associated with stomach cancer risk in the low-risk population of The Netherlands.
It is important to emphasize that our empirical score included most fresh vegetables and fruits, pulses, and black tea. Also, the ORs for high consumption of tea were associated with a reduction in risk (OR, 0.20; 95\% CI, 0.11-0.37). Previous studies conducted in China and Japan reported an important reduction in risk of stomach cancer for intake of green tea $[29,39,40]$. Also, a case-control study in Sweden showed that black tea intake was associated with a substantial decrease in gastric cancer risk [30]. On the other hand, a prospective study, carried out in The Netherlands, suggested that black tea intake did not affect the risk of gastric cancer [41]. It has been suggested that tea polyphenols could inhibit endogenous nitrosation [42]. Other mechanisms have been suggested; for example, an antiproliferative action, inhibition of cell transformation, and induction of apoptosis [42]. Further studies on the role of tea and coffee in gastric carcinogenesis are clearly of importance.

Salt (or salty food) has long been considered as a risk factor for gastric carcinoma and various previous studies $[6,11-13,15-16,19,22,32,43,44)$, have considered salt as an important risk factor for stomach cancer. This is probably due to a direct effect of salt on the gastric mucosa, leading to an atrophy [13]. Our mixed pattern showed positive loading for an important source of salt; that is, processed meat. Unexpectedly, this eating pattern was associated with a reduction in risk. A possible explanation could be related to the fact that the pattern also showed positive loading for eggs and pulses. These food groups were significantly protective in our study population. However, some foods rich in salt (salami, saucisson), frequently consumed in Uruguay, were associated with a significant increase in risk, as shown in the empirical risk enhancing score and in the analysis of food groups.

Previous studies of gastric cancer employed factor analysis in an effort to identify precise patterns which could be useful for preventive purposes. The study of Palli et al. [8], is noteworthy in that it identified a traditional pattern, associated with increased risk of gastric cancer, and a vitamin-rich pattern that was strongly protective. Our results are rather similar to those presented by Palli et al. [8].

There were significant differences by sex in our study. Similar findings were reported by McCullough et al. [35]. In fact, ten foods or food groups were statistically heterogeneous by sex. For example, total plant foods were strongly protective in men, whereas this food group was directly associated with gastric cancer in women. These differences could be related to reproductive factors [45]. It is rather difficult to exclude a chance finding [35]. Whatever the mechanisms involved, the reasons for these differences by sex are unclear. 
Our study, as occurs in other hospital-based casecontrol studies, has limitations. In the first place, the study is open to selection bias. This bias is almost impossible to avoid. We tried to minimize selection bias by frequency-matching of cases and controls for age, sex, residence, and urban/rural status. Classification bias is an other important bias. It is unlikely that misclassification bias could have occurred in our study. This is mainly due to the fact that the cases were mostly not informed about the dietary factors that could be operating in the process of gastric carcinogenesis. Nevertheless, recent changes in the diet could result in misclassification bias. In order to avoid this source of bias, we decided to ask about food consumption 5 years before the first symptom. It is acknowledged that is rather difficult to recall precisely the diet consumed in the past. In fact, some authors have suggested that casereferent studies are potentially prone to bias, and they have considered that only prospective studies are free from recall bias [46]. Interviewer bias is unlikely, because both our interviewers were unaware of the particular diets related to gastric cancer. Finally, a rather important limitation is linked with the impossibility of controlling according to Helicobacter pylori infection. Because infection with $H$. pylori is more prevalent in the less educated population, we included years of education in all models. On the other hand, the study has strengths. Perhaps the major strength is the high response rate, both for cases and controls.

In summary, the present study on food groups and risk of gastric cancer displayed consistent results arising from three different approaches: food group analysis, factor analysis, and analysis of empirical scores of risk. The following foods were directly associated with gastric cancer: processed (salted) meat, stewed meat, rice, white bread, potato, and winter squash. Processed meat and stewed meat are rich in salt; and grains, potato, and winter squash are an important source of starch. On the other hand, fresh vegetables, fruits, and black tea were strongly protective. Furthermore, fruits and vegetables are sources (among other protective agents) of ascorbic acid and carotenoids, which were associated with a high effect against the progression of gastric carcinogenesis. The role of black tea should be further investigated. The identification of these dietary patterns could allow the implementation of precise preventive strategies in order to avoid an increased incidence of gastric cancer in Uruguay. More precisely, diets rich in vegetables and fruits and with a low content of salty foods and starchy foods are recommendable.

Acknowledgments This study was supported by a grant from the International Agency for Research on Cancer, Lyon, France.

\section{References}

1. De Stefani E, Fierro L, Barrios E, Ronco A. Cancer mortality trends in Uruguay 1953-1991. Int J Cancer 1994;56:634-9.

2. Parkin DM, Whelan SL, Ferlay J, Raymond L, Young J. Cancer incidence in five continents, volume VII. IARC Scientific No. 148. Lyon: IARC; 1997.

3. Vassallo JA, Barrios E, De Stefani E, Ronco AL. II-Atlas de incidencia del cáncer en el Uruguay (in Spanish). Montevideo: Comisión Honoraria de Lucha contra el Cáncer; 2001.

4. Willett WC. Nutritional epidemiology. 2nd ed. Monographs in epidemiology and biostatistics, volume 30. Oxford: Oxford University Press; 1998.

5. Breslow NE, Day NE. Statistical methods in cancer research, volume 1. The analysis of case-control studies. IARC Scientific Publications No. 32. Lyon: IARC; 1980.

6. Harman HH. Modern factor analysis. 3rd ed. Chicago: University of Chicago Press; 1976.

7. Terry P, Hu FB, Hansen H, Wolk A. Prospective study of major dietary patterns and colorectal cancer risk in women. Am J Epidemiol 2001;154:1143-9.

8. Palli D, Russo A, Decarli A. Dietary patterns, nutrient intake and gastric cancer in a high-risk area of Italy. Cancer Causes Control 2001;12:163-72.

9. Manousos O, Day NE, Trichopoulos D, Gerovassilis F, Tzanou A, Polychonopoulou A. Diet and colorectal cancer: a case-control study in Greece. Int J Cancer 1983;32:1-5.

10. STATA. Stata reference guide. Release 6. Texas: College Station; 1999.

11. Graham S, Haughey B, Marshall J, Brasure J, Zielezny M, Freudenheim J, et al. Diet in the epidemiology of gastric cancer. Nutr Cancer 1990;13:19-34.

12. World Cancer Research Fund. Food, nutrition and the prevention of cancer: a global perspective. Washington DC: American Institute for Cancer Research; 1997.

13. Kono S, Hirohata T. Nutrition and stomach cancer. Cancer Causes Control 1996;7:41-55.

14. Segi M, Fukushima I, Fujisaku S, Kurihara M, Saito S. An epidemiological study on cancer in Japan. Gann 1957;48:1-63.

15. Haenszel W, Kurihara M, Segi M, Lee R. Stomach cancer among the Japanese in Hawaii. J Natl Cancer Inst 1972;49:96988.

16. Correa P, Fontham E, Pickle LW, Chen V, Lin Y. Dietary determinants of gastric cancer in South Louisiana inhabitants. J Natl Cancer Inst 1985;75:645-54.

17. La Vecchia C, Negri E, Decarli A, D’Avanzo B, Franceschi S. A case-control study of diet and gastric cancer in Northern Italy. Int J Cancer 1987;40:484-9.

18. Graham S, Schotz W, Martino P. Alimentary factors in the epidemiology of gastric cancer. Cancer 1972;30:922-38.

19. Risch HA, Jain M, Choi NW, Fodor JG, Pfeiffer CJ, Howe GR, et al. Dietary factors and the incidence of cancer of the stomach. Am J Epidemiol 1985;122:947-59.

20. Tuyns AJ, Kaaks R, Haelterman M, Riboli E. Diet and gastric cancer: a case-control study in Belgium. Int J Cancer 1992;51:16.

21. Nomura A, Grove JS, Stemmermann GN, Severson RK. A prospective study of stomach cancer and its relation to diet, cigarettes, and alcohol consumption. Cancer Res 1990;50:62731.

22. Kneller RW, McLaughlin JK, Bjelke E, Schuman LM, Blot WJ, Wacholder S, et al. A cohort study of stomach cancer in a highrisk American population. Cancer 1991;68:672-8.

23. Trichopoulos D, Ouranos G, Day NE, Tzonou A, Manousos O, Papadimitiou C, et al. Diet and cancer of the stomach: a casecontrol study in Greece. Int J Cancer 1985;36:291-7.

24. Jedrychowski W, Wahrendorf J, Popiela T, Rachtan J. A casecontrol study of dietary factors and stomach cancer in Poland. Int J Cancer 1986;37:837-42. 
25. Modan B, Lubin F, Barell V, Greenberg RA, Modan M, Graham $\mathrm{S}$. The role of starches in the etiology of gastric cancer. Cancer 1974:34:2087-92.

26. La Vecchia C, D'Avanzo B, Negri E, Decarli A, Benichou J. Attributable risks for stomach cancer in Northern Italy. Int J Cancer 1995;60:748-52.

27. Buiatti E, Palli D, Decarli A, Amadori D, Bianchi S, Biserni R, et al: A case-control study of gastric cancer and diet in Italy. Int J Cancer 1989;44:611-6.

28. Boeing H, Frentzel-Beyme R, Berger M, Berndt V, Göres W, Körner M, et al: Case-control study on stomach cancer in Germany. Int J Cancer 1991;47:858-64.

29. Kono S, Ikeda M, Tokudome S, Kuratsune M. A case-control study of gastric cancer and diet in northern Kyushu, Japan. Jpn J Cancer Res 1988;79:1067-74.

30. Hansson L-E, Nyrén O, Bergström R, Wolk A, Lidgren A, Baron J, et al. Diet and risk of gastric cancer. A population-based case-control study in Sweden. Int J Cancer 1993;55:181-9.

31. You W-C, Blot WJ, Chang Y-S, Ershow AG, An Q, Henderson $\mathrm{BE}$, et al: Diet and high risk of stomach cancer in Shandong, China. Cancer Res 1988;48:3518-23.

32. Correa P. Human gastric carcinogenesis: a multistep and multifactorial process. First American Cancer Society Award Lecture on Cancer Epidemiology and Prevention. Cancer Res 1992;52:673540.

33. Correa P, Fontham ETH, Bravo JC, Bravo LE, Ruiz B, Zarama $\mathrm{G}$, et al. Chemoprevention of gastric dysplasia: randomized trial of antioxidants and anti-Helicobacter pylori therapy. J Natl Cancer Inst 2000;92:1881-8.

34. Key TJ, Schatzkin A, Willett WC, Allen NE, Spencer EA, Travis RC. Diet, nutrition and the prevention of cancer. Public Health Nutr 2004;7:187-200.

35. McCullough ML, Robertson AS, Jacobs EJ, Chao A, Calle EE, Thun MJ. A prospective study of diet and stomach cancer mortality in United States men and women. Cancer Epidemiol Biomarkers Prev 2001;10:1201-5.

36. Hirayama T. A large scale cohort study on cancer risk by diet with special reference to the risk reducing effects of green-yellow vegetable consumption. In: Hayashi Y, Nagao M, Sugimura T, Tokoyama S, Tomatis L, Wattenberg LW, Wagen GN, editors.
Diet, nutrition and cancer: proceedings of the 16th International Symposium of the Princess Takamatsu Cancer Research Fund. Utrecht: Tokyo/VNU Science; 1986. pp. 41-53.

37. Hertog MGL, Bas Bueno-de-Mesquita H, Fehily AM, Sweetnam PM, Elwood PC, Kromhout D. Fruit and vegetable consumption and cancer mortality in the Caerphilly Study. Cancer Epidemiol Biomarkers Prev 1996;5:673-7.

38. Botterweck AAM, van den Brandt PA, Goldbohm RA. A prospective cohort study on vegetables and fruit consumption and stomach cancer risk in the Netherlands. Am J Epidemiol 1998; $148: 842-53$

39. Yu GP, Hsieh CC. Risk factors for stomach cancer: a populationbased case-control study in Shanghai. Cancer Causes Control 1991;2:169-74

40. Tajima K, Tominaga S. Dietary habits and gastro-intestinal cancers: a comparative case-control study of stomach and large intestinal cancers in Nagoya, Japan. Jpn J Cancer Res 1988;16:705-16.

41. Goldbohm RA, Hertog MGL, Brants HAM, van Poppel G, van den Brandt PA. Consumption of black tea and cancer risk: a prospective cohort study. J Natl Cancer Inst 1996;88:93-100.

42. Yang CS, Yang, G-Y, Chung JY, Lee M-J, Li C. Tea and tea polyphenols in cancer prevention. In: American Institute for Cancer Research, editor. Nutrition and cancer prevention. New insights into the role of phytochemicals. Advances in experimental medicine and biology, volume 492. New York: Kluwer Academic/ Plenum; 2001.

43. Nazario CM, Szklo M, Diamond E, Román-Franco A, Climent C, Suarez E, et al. Salt and gastric cancer: a case-control study in Puerto Rico. Int J Epidemiol 1993;22:790-7.

44. Chen VW, Abu-Elyazeed RR, Zavala DE, Ktsanes VK, Haenszel $\mathrm{W}$, Cuello C, et al. Risk factors of gastric precancerous lesions in a high-risk Colombian population. I. Salt. Nutr Cancer 1990;13: $59-65$.

45. La Vecchia C, D'Avanzo B, Franceschi S, Negri E, Parazzini F, Decarli A. Menstrual and reproductive factors and gastric-cancer risk in women. Int J Cancer 1994;59:761-4.

46. Feskanich D, Ziegler RG, Michaud DS, Giovannucci EL, Speizer FE, Willett WC, et al. Prospective study of fruit and vegetable consumption and risk of lung cancer among men and women. $\mathrm{J}$ Natl Cancer Inst 2000;92:1812-23. 